\title{
Solidification microstructure in a supercooled binary alloy
}

\author{
Hongfu Wang ${ }^{1, *}$, Cheng Tang ${ }^{1}$, Hongen $\mathrm{An}^{2}$, Yuhong Zhao ${ }^{3, \dagger}$ \\ ${ }^{1}$ School of Mechanical Engineering, North University of China, Taiyuan, Shanxi 030051, China \\ ${ }^{2}$ Faculty of Engineering, University Malaysia Sabah, Kota Kinabalu 88400, Malaysia \\ ${ }^{3}$ College of Materials Science and Engineering, North University of China, Taiyuan, Shanxi 030051, China
}

\begin{abstract}
The maximum undercooling that has been achieved for $\mathrm{Ni}-\mathrm{Cu}$ alloy, by using molten glass purification and cyclic superheating technology, is $270 \mathrm{~K}$. With the help of high-speed photography, the solidification front images of Ni-Cu alloy at various typical undercooling were obtained. Two grain refinements occurred in the range of $60 \mathrm{~K}<\Delta \mathrm{T}<100 \mathrm{~K}$ and $\Delta \mathrm{T}>170 \mathrm{~K}$, the solidification front became smoother, and the solidification position appeared randomly. With the increase of undercooling, the transition from solute diffusion to thermal diffusion leads to the transition from coarse dendrite to directional fine dendrite. At large undercooling, considerable stress is accumulated and some dislocations exist in the microstructure. However, the proportion of high-angle grain boundaries is as high as $89 \%$, with twin boundaries of $13.6 \%$ and most strain-free structures, and the microhardness decreases sharply. This indicates that the accumulated stress at large undercooling causes the plastic strains in the microstructure, and in the later stage of recalescence, part of the plastic strains is dissipated by the systemand acts as the driving force to promote the recrystallization of the microstructure.
\end{abstract}

Keywords: alloy, microstructure, rapid solidification, crystal growth

\section{Introduction}

Non-equilibrium solidification [1] is usually accompanied by a large undercooling, which enables metal or alloy melts to nucleate and grow at a faster solidificationvelocity. The microstructure has great differences compared with equilibrium solidification or near equilibrium solidification, which affects the plasticity, toughness, electrical conductivity, ductility, and other properties of materials. The deep undercooling rapid solidification technology in non-equilibrium solidification [2] can effectively remove impurities in the undercooled melt, which enables the undercooled melt to complete the solid-liquid transformation with a larger undercooling and accompanied by a faster solidification rate, thus obtaining excellent materials with special properties.

The solidification structure with fine grain and relatively uniform microstructure can be obtained by deep undercooling rapid solidification technology. In engineering, some materials with grain

\footnotetext{
* E-mail: wanghongfu@nuc.edu.cn

† E-mail: zhaoyuhong@nuc.edu.cn
}

refinement structures are also needed. The microstructure refinement in single-phase alloys is formed by spontaneous solidification in the melt. Its properties are very different from those of heattreated materials after cold deformation, so it has attracted extensive attention and become a typical research topic [3]. Generally speaking, the microstructure of single-phase alloys experiences the evolution of "dendrite - equiaxed grain - dendrite - equiaxed grain" at different undercooling. Two grain refinements occur at small and large undercooling, but the grain size and morphology of the two grain refinements are quite different. Liu [4], $\mathrm{Xu}[5,6]$, and $\mathrm{Lu}$ [7] found the above phenomena in different alloy studies. At present, many grain refinement mechanisms have been proposed to explain grain refinement, such as dynamic nucleation [8], dendrite remelting mechanism [9], critical velocity theory [10], recrystallization [11], etc. Grain refinement under small undercooling has been widely interpreted as the dendrite remelting mechanism, including dendrite remelting under solid-liquid interface tension proposed by Karma [12] and dendrite superheating remelt- 
ing proposed by Li [9]. A large number of experiments have shown that the dendrite superheating remelting mechanism can more fully explain the grain refinement at the small undercooling. However, there is no complete theoretical mechanism to explain the grain refinement at large undercooling, especially for high melting point alloys. In recent years, the stress-induced recrystallization mechanism [13] has attracted more attention. Liu [14], Liang [15], and $\mathrm{Xu}$ [16] studied the mechanism of Ni-Cu alloy. With the increase of undercooling, the solidification velocity increases continuously, and severe solidification shrinkage and thermal strain accumulate stress between the dendritic skeleton. When the accumulated stress exceeds the yield strength of the alloy, the dendrites undergo plastic deformation and fracture. In the later stage of recalescence, these plastic strains act as the driving force to promote recrystallization of the microstructure. At present, thegrain refinement mechanism at large undercoolingstill lacks reliable experimental validation, and stress-induced recrystallization has not been widely accepted.

$\mathrm{Ni}-\mathrm{Cu}$ alloy is a typical single-phase solid solution with infinite mutual solubility, which has good stability and is not easy to oxidize and burn out in the experimental process. And it is easy to obtain largeundercooling, so the alloy system is a good experimental object and Ni82Cu18 alloy is selected as the experimental object. In this article, it is aimed to set up the relationship among undercooling, microstructure transformation, and grain refinement in Ni82Cu18 alloy. Combined with BCT [17] model, electron backscattering diffraction (EBSD), transmission electron microscope (TEM), and microhardness, Ni82Cu18 alloy was simulated and characterized. The microstructure transformation mechanism in binary single-phase alloy was systematically explained, which further provided a reliable basis for grain refinement mechanism at large undercooling.

\section{Methods}

Ni82Cu18 alloy was prepared by melting pure nickel particles (99.99\%) and pure copper particles $(99.99 \%)$ in a vacuum arc melting furnace under the protection of argon. The alloy was remelted at least three times to ensure the uniformity of chemical composition, and a $4 \mathrm{~g}$ sample was cut from each original ingot for experiments. The alloy sample and quartz tube were cleaned in an ultrasonic cleaner for $10 \mathrm{~min}$ to achieve the preliminary purification effect. Then the quartz tube was installedin the center of the induction coil containing the alloy sample and $2 \mathrm{~g}$ of anhydrous $\mathrm{B}_{2} \mathrm{O}_{3}$. The entire melting process was carried out in a vacuum chamber, which was evacuated to a pressure of $3 \times 10^{-3} \mathrm{~Pa}$ and pure Ar gas was backfilled to a pressure of $5 \times 10^{-2} \mathrm{~Pa}$. First, the temperature was increased to $800^{\circ} \mathrm{C}$ to fully melt $\mathrm{B}_{2} \mathrm{O}_{3}$. When there were no bubbles and the system was stable, the temperature was further increased to 100-250 $\mathrm{K}$ higher than the melting point of the alloy and held for about $20 \mathrm{~min}$ to further purify the system. The alloy was first superheated and then cooled down cyclically until an ideal undercooling was obtained. In the whole experiment, the temperature was recorded by an infrared thermometer with a response time of $1 \mathrm{~ms}$, and the solidification process during recalescence was captured by a high-speed camera (OLYMPUS I-speed3).

The solidified samples were prepared for metallurgical analysis. After cutting, setting, and polishing, the undercooled sample was etched with a $50 \% \mathrm{HNO}_{3}$ aqueous solution. The microstructure of the sample was observed by optical microscopy (Olympus GX71). The samples were polished with $\mathrm{Al}_{2} \mathrm{O}_{3}$ colloidal suspension on a vibratory polisher and the grain and boundary characteristics were observed by EBSD. The EBSD experiment was carried out in a Vega II XMH scanning electron microscope equipped with an automatic EBSD analysis system, and the scanning step was approximately $1 / 5$ to $1 / 10$ of the average grain size of the sample microstructure. This experiment studies the relative orientation of grains rather than the absolute orientation, and so there are no special requirements for the placement of samples in the analysis process. After mechanical grinding and ion grinding, the dislocations and other defects in the microstructure were observed by TEM (TecnaiFG2). The microhardness of each sample was measured on the microhardness tester (HMV-2T) with a load of $2.95 \mathrm{~N}$ 


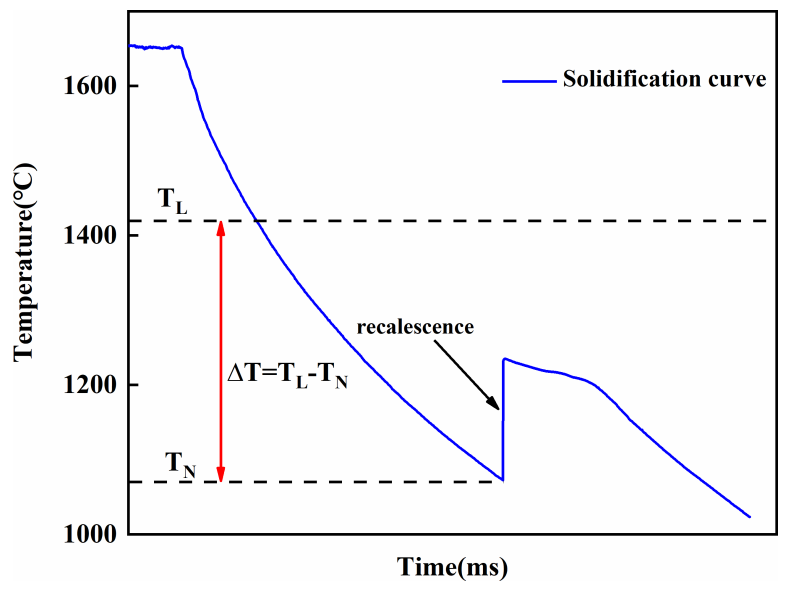

Fig. 1. Undercooling process and calculation of undercooling.

for $15 \mathrm{~s}$. Each sample was tested at least 20 points across the surface and the average value was taken as experimental data.

The experiment is an undercooling process of natural nucleation. $\mathrm{B}_{2} \mathrm{O}_{3}$ purifier is used to remove impurities from the alloy liquid. Once natural cooling is adopted, the alloy liquid will begin to nucleate and solidify rapidly at a lower temperature. This is a spontaneous process, and the rapid solidification stage is not controlled by heat dissipation conditions. The calculation of undercooling is reflected in the solidification curve in Figure 1.

BCT model can be used to explain the microstructure and morphology transformation process of the experimental alloy. The BCT model divides the initial undercooling into four components. The initial undercooling of the alloy melt is composed of the following four components:

$$
\Delta T=\Delta T r+\Delta T c+\Delta T k+\Delta T t
$$

where $\Delta T r$ is curvature undercooling, $\Delta T c$ is solute undercooling, $\Delta T k$ is kinetic undercooling, and $\Delta T t$ is thermal undercooling.

\section{Results and discussions}

\subsection{Microstructure transformation}

The maximum undercooling has achieved $270 \mathrm{~K}$ for Ni82Cu18 alloy by using molten glass purification combined with cyclic superheating

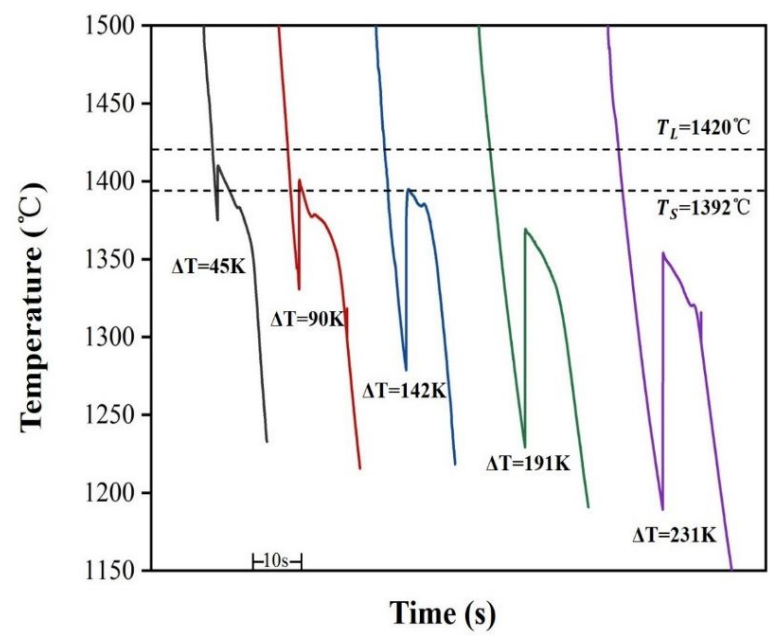

Fig. 2. The recalescence process of Ni82Cu18 alloy under different undercooling.

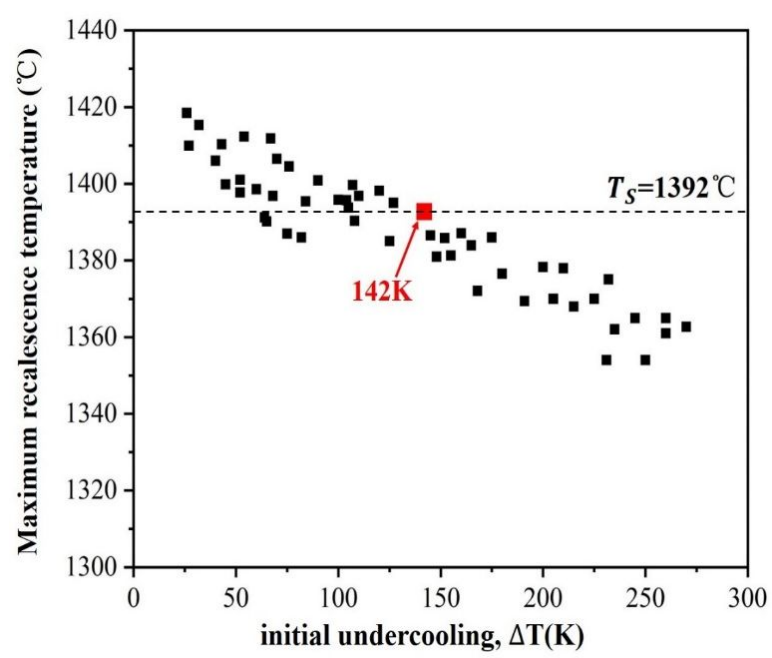

Fig. 3. Maximum recalescence temperature of Ni82Cu18 alloy under different undercooling.

technology. The infrared thermometer is used to record the temperature change of the whole alloy liquid during solidification and recalescence. Figure 2 shows the recalescence processes under several typical undercooling, and Figure 3 shows the maximum recalescence temperatures under different undercooling.

Figures 2 and 3 show that with the increase of undercooling, there is a gradual decrease in the rapid solidification time, the maximum recalescence temperature, and the slow solidification time 
after recalescence. When the undercooling is large enough, the slow solidification process can hardly be seen. $\mathrm{Li}$ [9] pointed out in the dendrite superheating remelting mechanism that the latent heat of crystallization released during rapid solidification at small undercooling makes the temperature rise above the solid line and the primary dendrites remelt and refine due to superheat. We can see that the first grain refinement occurs in Ni82Cu18 alloy at $\Delta \mathrm{T}=76 \mathrm{k}$, and the microstructure is filled with a large number of fine equiaxed grains, as shown in Figure 5c.

The solid-phase temperature of Ni82Cu18 alloy is $1,392^{\circ} \mathrm{C}$. When $\Delta \mathrm{T}=142 \mathrm{~K}$, the maximum recalescence temperature just reaches the solidphase line temperature (Figure 2), which indicates that the critical hypercooling is just reached at this time [18]. When $\Delta \mathrm{T}>142 \mathrm{~K}$, the maximum recalescence temperature does not exceed the solidphase line temperature (Figure 3), and the latent heat of crystallization released during the solidification process is not enough to make the dendrites remelted. However, when $\Delta \mathrm{T}>170 \mathrm{~K}$, the second grain refinement occurs in Ni82Cu18 alloy, and a large number of fine equiaxed grains appear again in the microstructure, as shown in Figure 5h. This indicates that grain refinement at this time is not related to dendrite remelting. At present, the stressinduced recrystallization mechanism has attracted much attention. The grain refinement mechanism at large undercooling is further demonstrated below.

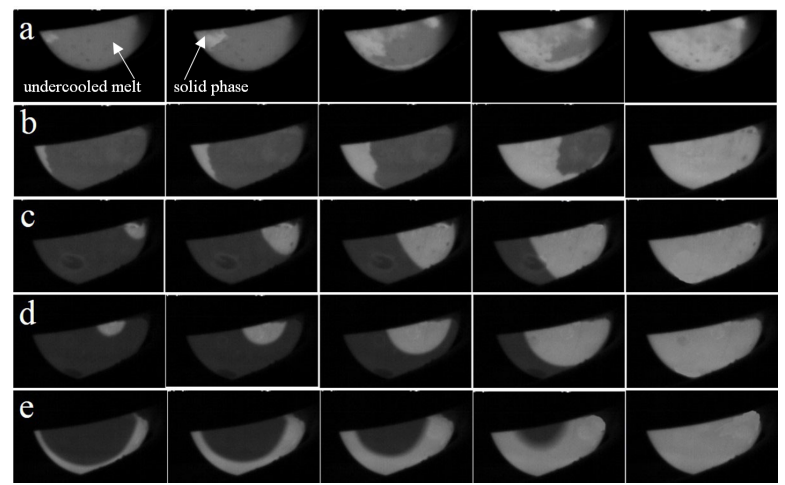

Fig. 4. High-speed video camera images of Ni82Cu18 alloy with different undercooling degrees (A) $60 \mathrm{~K}$; (B) $140 \mathrm{~K}$; (C) $194 \mathrm{~K}$; (D) $220 \mathrm{~K}$; (E) $250 \mathrm{~K}$.
The heat released by the alloy during rapid solidification is converted into light signals, which can be captured using a high-speed video camera. The solidification process can be effectively presented by using the brightness of the solidification interface in the image [19]. The continuous solidification process of Ni82Cu18 alloy under different undercooling is shown in Figure 4, where the bright areas represent the condensed solids and the dark areas represent the undercooled melts. At small undercooling, the solidification process starts at the interface where contact is established between the alloy melt and $\mathrm{B}_{2} \mathrm{O}_{3}$, and the solidification front is a plane with a small angle, as shown in Figure 4a, b. Since most of the impurities are distributed at the interface and the quartz tube wall, the solidification velocity at the interface and on the quartz tube wall is obviously faster than that inside the undercooled melt. With the increase of the undercooling, the purification effect becomes gradually obvious. The solidification front becomes very smooth and the solidification position appears randomly, as shown in Figure 4c-e, and the phenomenon of nonuniform solidification velocity does not exist in different positions. We can see from Figure 4 that the solidification of the alloy always starts at one point and migrates from one side to the other, rather than crystallization growth at multiple locations. With the increase of undercooling, the solidification front becomes smooth, the solidification position is not fixed, and the solidification velocity of each part becomes uniform gradually.

By analyzing the microstructure morphology of all samples, we found that the following transformation process exists in the microstructure of Ni82Cu18 alloy, as shown in Figure 5.The grain size of Ni82Cu18 alloy under different undercooling was studied systematically by drawing lines and measuring dendritic trunk length, as shown in Figure 6 . Therewere four characteristic ranges in undercooled Ni82Cu18 alloy:

1. $\Delta \mathrm{T}<60 \mathrm{~K}$. When the undercooling is in the range of $0-60 \mathrm{~K}$, the microstructure is occupied by coarse dendrites surrounded by the remelted secondary dendrite arms, and there is no obvious directivity between the den- 

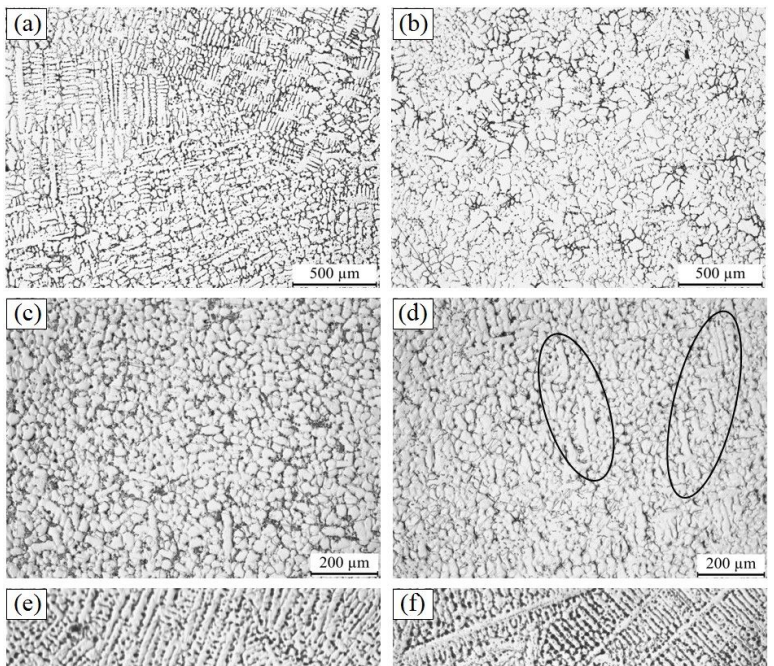

(3).
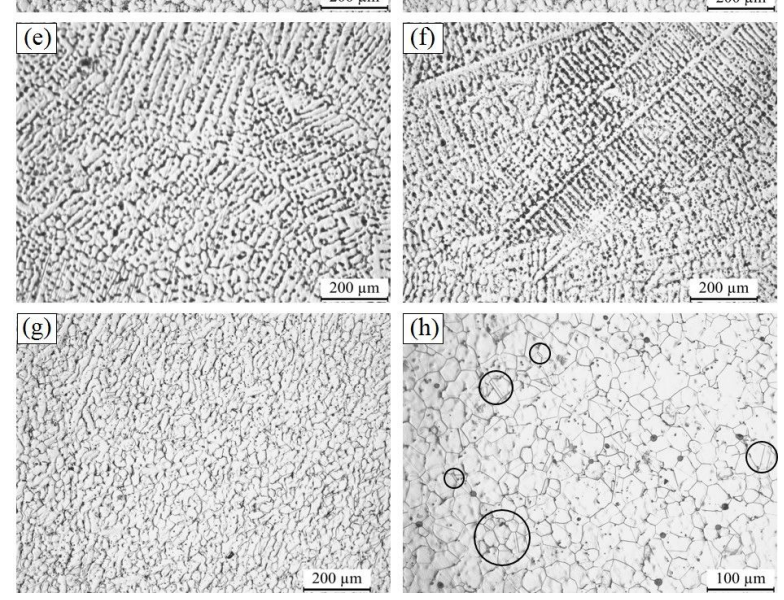

Fig. 5. Microstructure of Ni82Cu18 alloy at different undercooling. (A) $30 \mathrm{~K}$; (B) $60 \mathrm{~K}$; (C) $76 \mathrm{~K}$; (D) $100 \mathrm{~K}$; (E) $120 \mathrm{~K}$; (F) $155 \mathrm{~K}$; (G) $170 \mathrm{~K}$; and $(\mathbf{H}) 210 \mathrm{~K}$.

drites, as shown in Figure 5a. The grain size in this undercooling range is very large, as shown in Figure 6.

2. $60 \mathrm{~K}<\Delta \mathrm{T}<100 \mathrm{~K}$. When the undercooling is in the range of $60-100 \mathrm{~K}$, fine equiaxed grains gradually appear in the microstructure, and the grain size decreases to about $53 \mu \mathrm{m}$, as shown in Figure 6. When $\Delta \mathrm{T}=$ $60 \mathrm{~K}$, fine equiaxed grains begin to appear in the microstructure (Figure 5b), which indicates that starting from $\Delta \mathrm{T}=60 \mathrm{~K}$, the dendrites in the microstructure begin to break into equiaxed grains, and the first grain refinements [19] begin to occur. When $\Delta \mathrm{T}=$ $76 \mathrm{~K}$, the microstructure is all composed of fine equiaxed grains with relatively straight

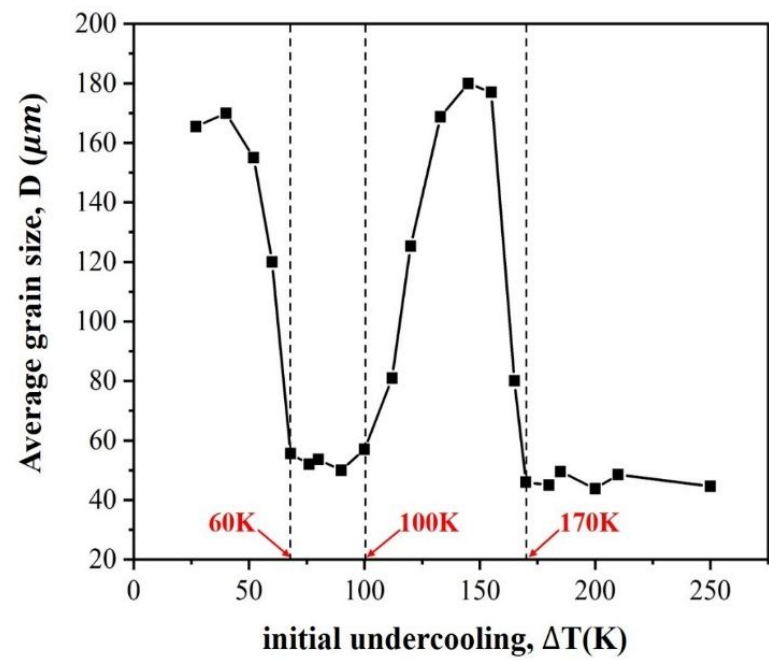

Fig. 6. Evolution of grain size of Ni82Cu18 alloy under different undercooling.

grain boundaries (Figure $5 \mathrm{c}$ ). When $\Delta \mathrm{T}=$ $100 \mathrm{~K}$, the dendrites appear again in the microstructure (circled), and the number of equiaxed grains decreases gradually (Figure 5d), which indicates that the first grain refinement has ended gradually at $\Delta \mathrm{T}=$ $100 \mathrm{~K}$.

3. $100 \mathrm{~K}<\Delta \mathrm{T}<170 \mathrm{~K}$. With the increase of undercooling to a level between $100 \mathrm{~K}$ and $170 \mathrm{~K}$, the equiaxed grains gradually disappear and the microstructure is welldeveloped directional fine dendrites (Figure $5 \mathrm{e}, \mathrm{f}$ ). However, the dendrite morphology in this range is completely different from that in the small undercooling, which indicates that the dendrite growth mechanism has been changed.

4. $\Delta \mathrm{T}>170 \mathrm{~K}$. When $\Delta \mathrm{T}=170 \mathrm{~K}$, directional fine dendrites begin to disappear. Deformed grains, fractured dendrites, and equiaxed grains with straight grain boundaries exist in the microstructure, and the grain boundaries migrate significantly (Figure 5g), which indicates the second grain refinement begins to occur in the microstructure. Yang [15] also found this phenomenon in other alloys. When $\Delta \mathrm{T}=210 \mathrm{~K}$, the microstructure is wholly composed of hexagonal equiax- 

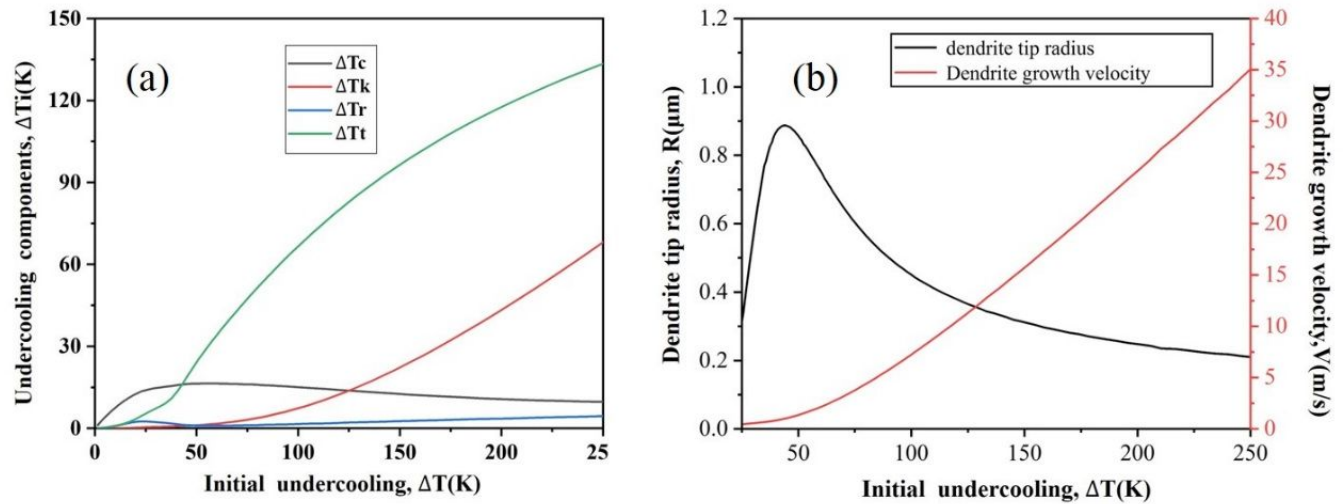

Fig. 7. (A) Evolution of component undercooling with initial undercooling. (B) Evolution of dendrite tip radius and dendrite growth velocity with initial undercooling.

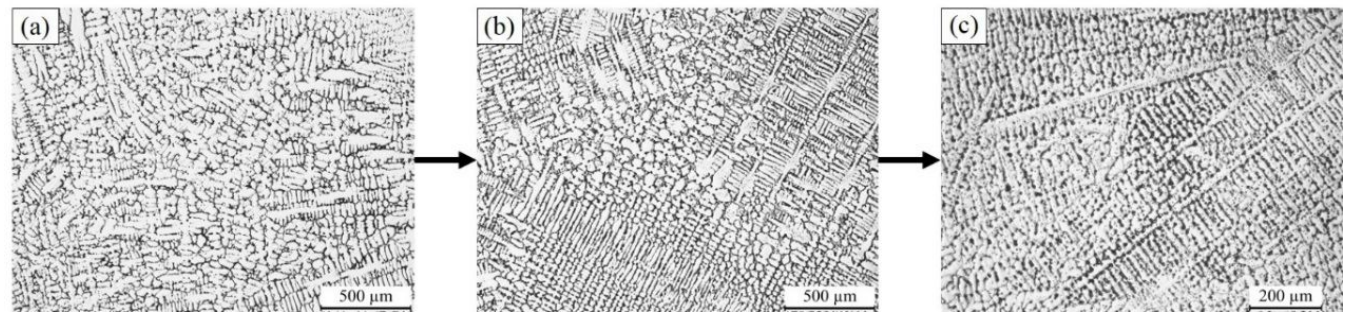

Fig. 8. Dendrite morphology at different undercooling (A) $25 \mathrm{~K}$; (B) $45 \mathrm{~K}$; (C) $150 \mathrm{~K}$.

ial grains with straight grain boundaries, and there are a large number of annealing twins (circled), as shown in Figure 5h. The grain size of the microstructure decreases to about $48 \mu \mathrm{m}$ (Figure 6).

It can be seen from Figures 5 and 6 that two grain refinements occur in Ni82Cu18 alloy at different undercooling. The microstructural transformation of "coarse dendrite - equiaxed grain - directional fine dendrite - equiaxed grain" occurred in Ni82Cu18 alloy in the undercooling range. Compared with the first grain refinement, the grain boundary of equiaxed grain in the second grain refinement is straighter and the grain size is smaller, and there are annealing twins that the former does not have. This may be due to more accumulated stress at large undercooling, which causes recrystallization of the structure at the later stage of solidification [13]. The dendrite morphology in the range of $100 \mathrm{~K}<\Delta \mathrm{T}<170 \mathrm{~K}$ is also quite different from that in the small undercooling. In the following sections, the microstructure transformation mechanism is further explained based on the BCT model, EBSD technique, TEM characterization, and microhardness.

\subsection{Dendrite growth in solidification}

The solidification microstructure of alloys with different undercooling degrees is quite different. The solidification microstructure of the alloy with small undercooling shows coarse dendrites, while the microstructure with large undercooling is very fine directional dendrites. This indicates that the factors controlling dendrite growth at different undercooling have changed. By analyzing the evolution process of different components against the initial undercooling, the microstructure transformation during the dendrite precipitation can be effectively explained. Figure 7 shows the evolution of component undercooling, dendrite tip radius, and dendrite growth velocity according to the BCT model, taking place resultant to the initial undercooling in Ni82Cu18 alloy. 

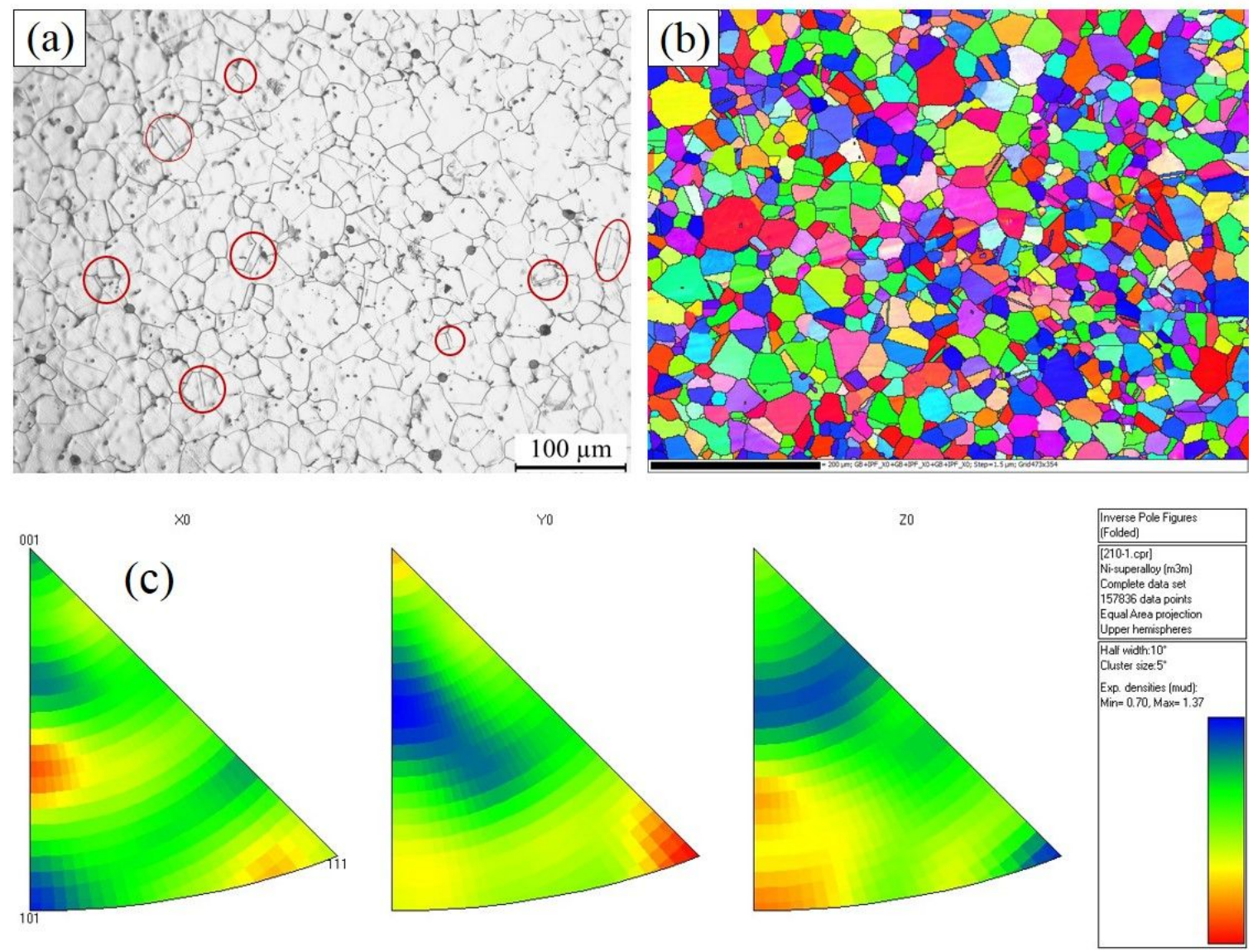

Fig. 9. (A) Microstructure of Ni82Cu18 alloy at $210 \mathrm{~K}$; (B) grain orientation of Ni82Cu18 alloy at $210 \mathrm{~K}$; (C) the inverse pole figure of (B).

As shown in Figure $7 \mathrm{a}$, when $\Delta \mathrm{T}<40 \mathrm{~K}$, the solute undercooling $\Delta T c$ is greater than the other three. This indicates that the dendrite growth of Ni82Cu18 alloy is mainly affected by solute diffusion, resulting in a very slow dendrite growth velocity (Figure $7 \mathrm{~b}$ ). In the range of $0-40 \mathrm{~K}$, the solute undercooling gradually increases and the dendrite tip radius also increases. This directly results in the coarse-dendrite microstructure (Figure 8a). In the range of 40-60 K, the thermal undercooling $\Delta T t$ gradually exceeds the solute undercooling $\Delta T c$, but the $\Delta T t$ and $\Delta T c$ are still in the same order of magnitude, which indicates that both thermal and solute diffusion control the dendrite growth of Ni82Cu18 alloy in this range. When $\Delta \mathrm{T}=48 \mathrm{~K}$, there is a maximum value of solute undercooling $\Delta T c$, so the dendrite tip radius also increases to a maximum value. Therefore, in the range of $40 \mathrm{~K}<$
$\Delta \mathrm{T}<60 \mathrm{~K}$, the dendrite growth velocity is still relatively slow and the dendrites in the microstructure are not only coarser but also more directional, as shown in Figure 8b.

As the undercooling continues to increase, the thermal undercooling $\Delta T t$ and kinetic undercooling $\Delta T k$ rapidly increase, resulting in a rapid decrease of the dendrite tip radius and a rapid increase of the dendrite growth velocity. When $\Delta \mathrm{T}$ $>100 \mathrm{~K}$, thermal diffusion plays a dominant role in dendrite growth, and the microstructure is welldeveloped directional dendrites (Figure 8c). When $\Delta \mathrm{T}>170 \mathrm{~K}$, the dendrite tip radius $\mathrm{R}$ does not decrease basically, but dendrites have been replaced by equiaxed grains in microstructure, as shown in Figure $5 \mathrm{~g}$, h. The following part will further explore the microstructure transformation mechanism at large undercooling. 

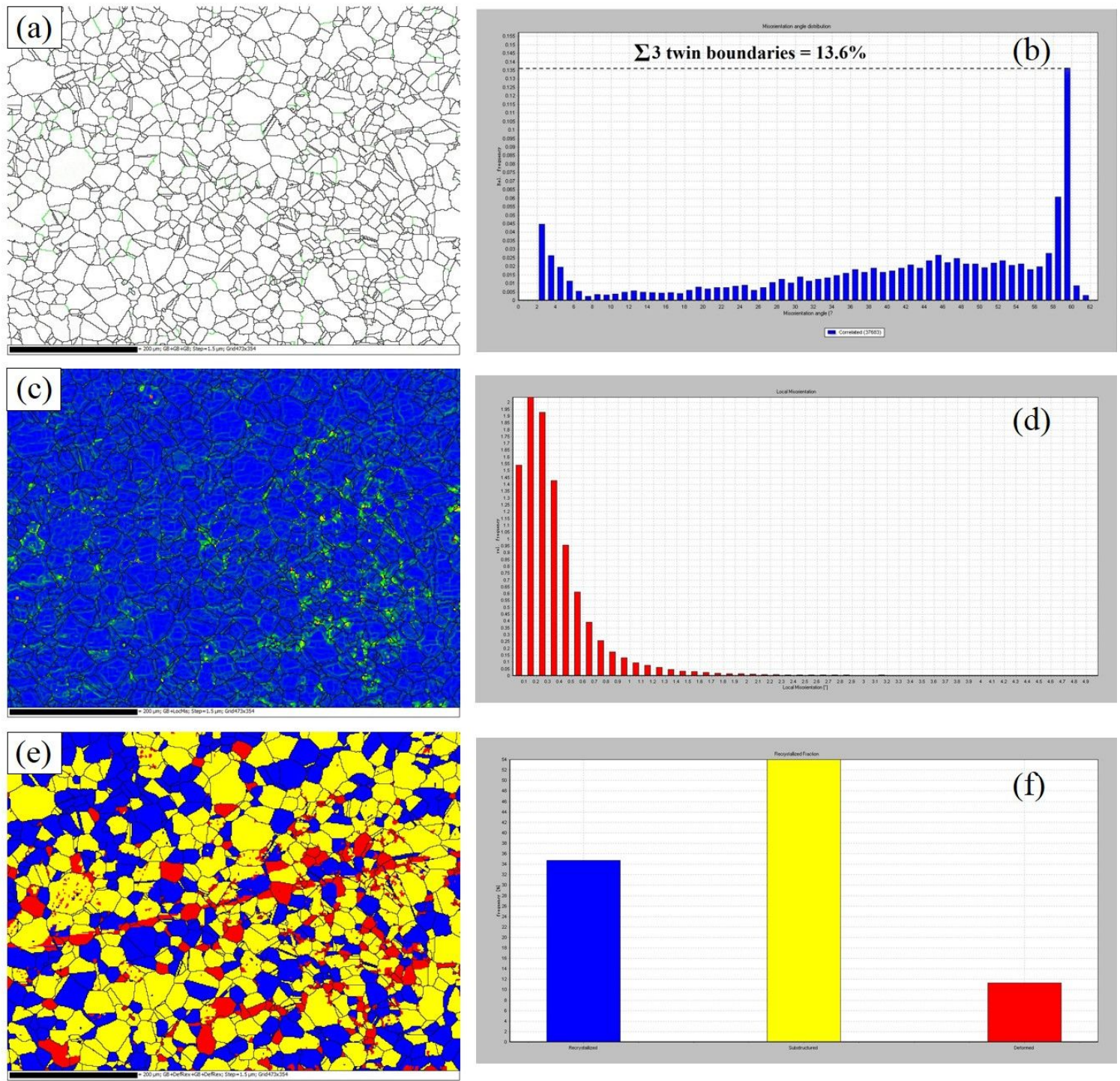

Fig. 10. (A) Grain boundaries of Figure 9b; (B) misorientation angle distribution of (A); (C) local misorientation of Figure 9b; (D) local misorientation distribution of (C); (E) recrystallization distribution of Figure 9b (blue is recrystallization, yellow is substructure and red is a deformed grain); (F) recrystallized fraction of (E).

\subsection{Grain refinement at large undercool-} ing

The stress-induced recrystallization mechanism [13] indicates that the accumulated stress at large undercooling causes plastic strain in the microstructure. The plastic strain as the driving force causes recrystallization of the solidified structure in the late stage of recalescence. EBSD technology was used to characterize the microstructure at
$210 \mathrm{~K}$ to obtain the orientation information and grain boundary characteristics of grains, as shown in Figures 9 and 10.

Recrystallization, driven by stored energy, replaces the original deformed grains by nucleation and growth of new grains. Therefore, under the driving force, the grain boundaries must have migrated. After recrystallization, the crystallographic orientations of adjacent grains must be different, with high-angle grain boundaries and stable an- 
nealing twins, and this has been confirmed in the study by Wang [16]. It can be seen from Figure 9b, $\mathrm{c}$ that the grain refinement does not introduce new texture, and the microstructure presents random distribution. Figure 10a shows the corresponding grain boundary character distribution. The highangle grain boundaries are black (the misorientation angle is $>15^{\circ}$ ) and the small-angle grain boundaries are green (the misorientation angle is in the range of $2^{\circ}-15^{\circ}$ ). At large undercooling, the proportion of high angle grain boundaries is about $90 \%$, and the proportion of twin boundaries reaches $13.6 \%$ (Figure 10b). The local misorientation can qualitatively determine the deformation degree and dislocation density of the microstructure, as shown in Figure 10c. Large areas of blue and small amounts of green and red indicate that the microstructure contains a small amount of dislocations. Most of the values in local misorientation distribution (Figure 10d) are small, which also indicates the low plastic strain in the microstructure. Figure $10 \mathrm{e}$, f can more intuitively represent the recrystallization distribution. Where the recrystallized grains account for $35 \%$, the substructures with a few dislocations exceed $50 \%$, and the deformed grains are only $11 \%$.

In the microstructure of $\Delta \mathrm{T}=210 \mathrm{~K}$, highangle grain boundaries and twin boundaries are in high proportion. The deformation degree of the microstructure is low, and most of the microstructure characterized by deformation is substructures with a few dislocations and recrystallized grains with stability and low energy. This indicates that the recovery and recrystallization have occurred in the microstructure after the critical undercooling [17].

According to the stress-accumulation model [13], the primary solid fraction is higher at large undercooling, and the interaction between flowing melt and primary dendrites results in stress accumulation. When the stress exceeds the yield strength of the alloy, the plastic strains occur in the dendrites and the microstructure is in an unstable and high-energy state. At the late stage of recalescence, the migration of grain boundaries has occurred in the microstructure, driven by the stored deformation energy, and new undistorted equiaxed grains are formed gradually. The solid fraction in the recalescence process is:

$$
f_{S}^{R}=\frac{C_{P}}{\Delta H}\left(T_{R}-T_{n}\right)
$$

The stress accumulation can be expressed as follows:

$$
\begin{aligned}
\sigma_{S}= & \frac{160 \mu \cdot|\vec{a}|}{\left(f_{R}^{S}\right)^{2} t_{f} \lambda_{2}^{2}} \times \frac{\left(1-f_{S}(\vec{x})\right)}{f_{S}(\vec{x})} \\
& \left\{\begin{array}{c}
|\vec{a}| \cdot \beta_{S}\left[\begin{array}{c}
f_{S}(\vec{x})-f_{c o h}+\frac{1}{\left(1-f_{S}\right)}-\frac{1}{\left(1-f_{c o h}\right)} \\
+2 \ln \left[\frac{1-f_{S}(\vec{x})}{1-f_{c o h}}\right]
\end{array}\right] \\
-\frac{2\left(1+\beta_{S}\right) \alpha_{t h e r} \Delta T_{0} \Delta T}{3 G_{S}} Q\left[f_{S}(\vec{x})\right]
\end{array}\right\}
\end{aligned}
$$

The evolution of solid fraction and stress accumulation with initial undercooling of Ni82Cu18 alloy according to Eqs (2) and (3) is shown in Figure 11a, b.

When the undercooling is small, the solidification velocity is small, the solid fraction is small, and the rapid solidification process cannot form the continuous dendrites network, so the stress cannot be accumulated between dendrites. When $\Delta \mathrm{T}=80 \mathrm{~K}$, the primary solid fraction approaches $30 \%$, and the continuous dendrites network began to form. The flowing melt and dendrite began to interact to accumulate stress between dendrites, as shown in Figure 11b. With the increase of solid fractions, the accumulated stress also increases. After critical undercooling $\Delta \mathrm{T}^{*}$, the stress increases almost linearly. At the large undercooling, there is the larger stress in the microstructure (Figure 11b), while the microhardness drops sharply after the critical undercooling (Figure 12a), and a large number of hexagonal equiaxed grains appear in the microstructure (Figure 12b). This indicates that at large undercooling, the microstructure is in a stable state with low energy, and the plastic strains caused by stress as stored deformation energy promote the recrystallization of the microstructure, which can also be found in the rapid quenching and hypercooling alloy experiment of Yang [15], which concerns the undercooling experiment. This is strong evidence for "stress-induced recrystallization." 

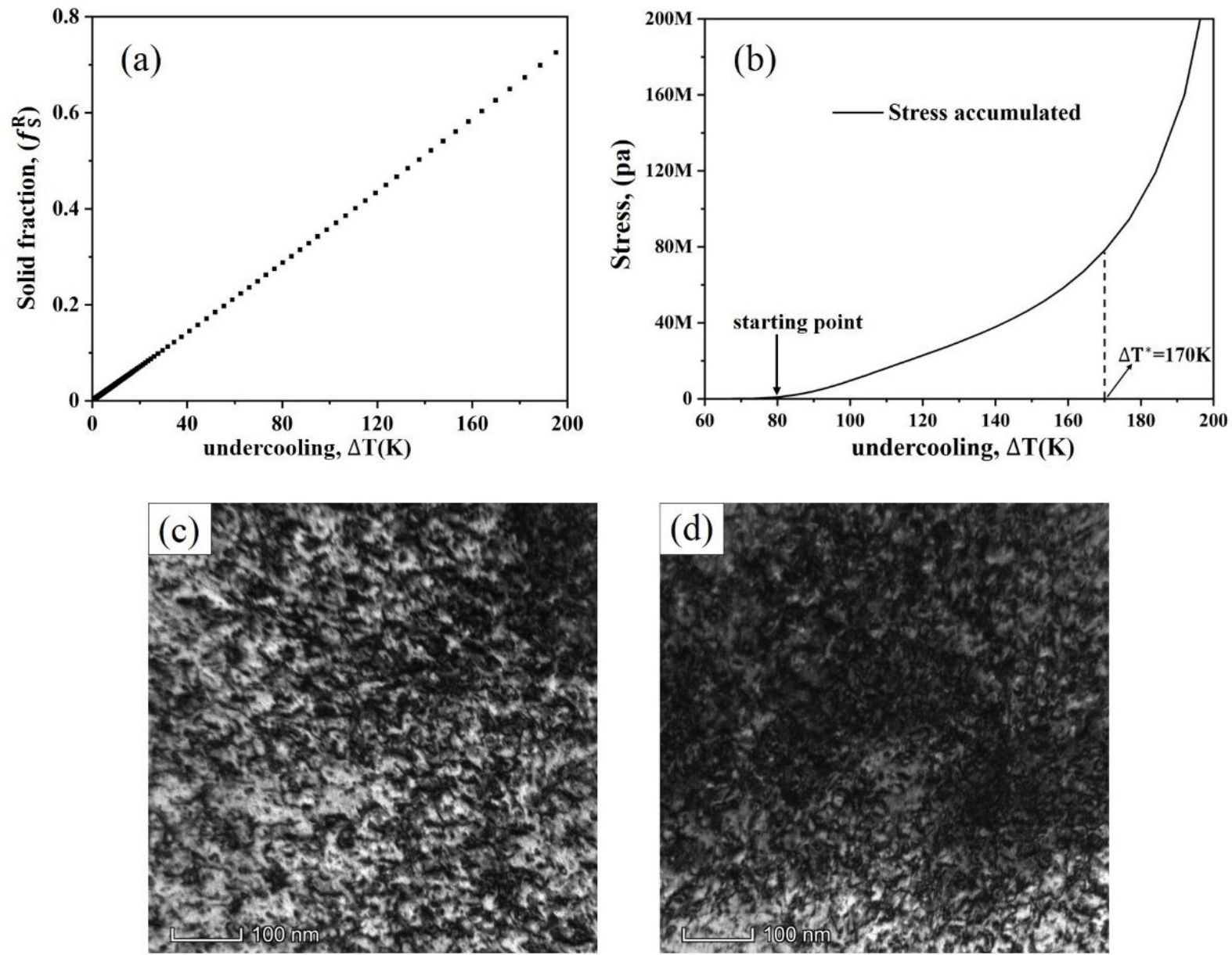

Fig. 11. (A) The solid fraction at different undercooling; (B) Stress at different undercooling; (C, D) TEM bright field image of Ni82Cu18 alloy at 270 K.TEM, transmission electron microscope.

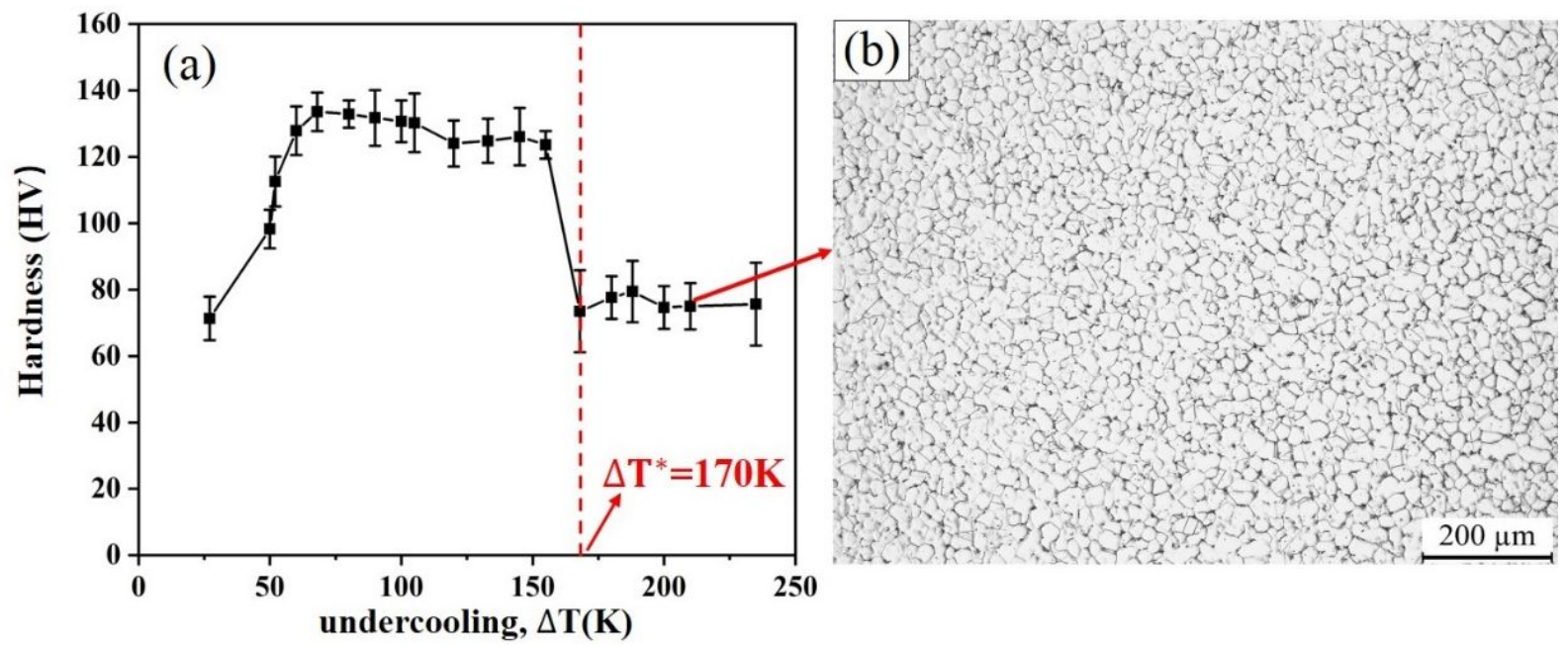

Fig. 12. (A) Hardness of Ni82Cu18 alloy at different undercooling; (B) Microstructure of Ni82Cu18 alloy at $210 \mathrm{~K}$. 


\section{Conclusions}

Various analyses were carried out on the solidified undercooled samples of Ni88Cu12 alloy. The main conclusions are as follows:

1. Two grain refinements occur in $\mathrm{Ni82 \textrm {Cu } 1 8}$ alloy within the undercooling range, on the whole facilitating the microstructure transformation into"coarse dendrite - equiaxed grain - directional fine dendrite - equiaxed grain."

2. At small undercooling, the solidification front is an interface with a small angle and the solidification velocity at the melt interface is faster than that inside. With the increase of undercooling, the solidification velocity of all parts of the melt becomes more uniform, and the solidification front becomes smoother and appears randomly.

3. In the dendrite growth, the dendrites controlled by solute diffusion are coarse dendrites. The dendrites controlled by solute diffusion and thermal diffusion are directional coarse dendrites. The dendrites controlled by thermal diffusion are directional fine dendrites.

4. In the solidification structure with a large undercooling degree, most of them are equiaxed grains and subgrains, and there are also a few deformed grains. The equiaxed grains contain a high proportion of largeangle grain boundaries and annealing twins. The microhardness also drops sharply after the critical undercooling. This indicates that extensive recovery and recrystallization have occurred and further proves the correctness of stress-induced recrystallization.

$\mathrm{Ni}-\mathrm{Cu}$ alloy is a typical binary ideal experimental object. Its microstructure evolution process and grain refinement mechanism have important reference significance in the research of other binary alloy systems and even multicomponent alloy systems. One of the future research directions that can be pursued is multicomponent alloys, the objective being to observe whether there is grain refinement and to explore its grain refinement mechanism. The question of whether there to opt for a new grain refinement mechanism or the coupling of existing grain refinement mechanisms is worthy of further study, which requires the further efforts of the majority of researchers.

\section{Funding}

The study was funded by the National Natural Science Foundation of China(Nos. 51701187, 51674226) and the Basic Applied Research Projects in Shanxi Province (201801D221151).

\section{Conflict of interest}

The author declares that there exists no conflict of interest.

\section{References}

[1] Herlach DM. Non-equilibrium solidification of undercooled metallic melts. Key Eng Mater. 1993;81:83-94.

[2] Castle EG, Mullis AM, Cochrane RF. Mechanism selection for spontaneous grain refinement in undercooled metallic melts. ActaMaterialia. 2014;77:76-84.

[3] Liu N, Liu F, Yang G, Chen Y, Chen D, Yang C, et al. Grain refinement of undercooled single-phase Fe70Co30 alloys. Phys B. 2007;387:151-5.

[4] Xu XL, Chen YZ, Liu F. Recrystallization mechanism of grain refinement in hypercooled single phase alloys. JCryst Growth. 2013;377:153-9.

[5] Lu SY, Li JF, Zhou YH. Grain refinement in the solidification of undercooled Ni-Pd alloys. JCryst Growth. 2007;309:103-11.

[6] Horvay G. The tension field created by a spherical nucleus freezing into its lessdense undercooled melt. Int J Heat Mass Transfer. 1965;8:195-243.

[7] Li JF, Lü YL, Yang GC, Zhao YH. Directional solidification of undercooled melt. Prog Nat Sci. 1997;7:98-103.

[8] Willnecker R, Herlach DM, Feuerbacher B. Grain refinement induced by a critical crystal growth velocity in undercooled melts. Appl Phys Lett. 1990;56:324-6.

[9] Xu XL, Liu F. Recrystallization and twinning in rapidly solidified nickel based alloys without man-made plastic deformation. J Alloys Compd. 2014;615:156-62.

[10] Karma A. Model of grain refinement in solidification of undercooled melts.Int $\mathbf{J}$ Non-Equilibrium Process. 1998;11:201-33.

[11] Liu F, Gencang Y. Stress-induced recrystallization mechanism for grain refinement in highly undercooled superalloy. JCryst Growth. 2001;231:295-305.

[12] Liu F, Yang GC. Rapid solidification of highly undercooled bulk liquid superalloy: recent developments, future directions. Int Mater Rev. 2006;51:145-70.

[13] Boettinger WJ, Coriell SR, Trivedi R. In: Mehrabian $\mathrm{R}$, Parrish PA editors. Rapid solidification processing: principles and technologies IV. Baton Rouge, LA: Claitor'sPulishing Division; 1988. pp. 13-18. 
[14] Zhang T, Liu F, Wang HF, Yang GC. Grain refinement in highly undercooled solidification of Ni85Cu15 alloy melt: Direct evidence for recrystallization mechanism. ScriptaMaterialia. 2010;63:43-6.

[15] Yang W, Liu F, Liu H, Wang HF, Yang GC, Zhou YH. Numerical description for the recalescence of bulk-undercooled Cu70Ni30 alloy. JCryst Growth. 2009;311:3225-30.

[16] Wang HF, FengLI, KangWA, Zhai HM. Effect of back diffusion on overall solidification kinetics of undercooled single-phase solid-solution alloys. Trans Nonferrous Metals Society of China. 2012;22:642-6.
[17] $\mathrm{Xu} \mathrm{XL}$, Chen YZ, Liu F. Evidence of recrystallization mechanism of grain refinement in hypercooled Co80Pd20 alloys. Mater Lett. 2012;81:73-5.

[18] Yang W, Xu ZF, Li WJ, Cai CC, Li S, Liu F, et al. Comparisons of grain refinement and recalescence behavior during the rapid solidification of undercooled $\mathrm{Cu}-\mathrm{Co}$ and $\mathrm{Cu}-\mathrm{Ni}$ alloys. Phys B. 2011;406:3710-4.

[19] Xu XL, Liu F. Crystal growth due to recrystallization upon annealing rapid solidification microstructures of deeply undercooled single phase alloys quenched before recalescence. Cryst Growth Des. 2014;14:2110-4.

Received 2021-06-18

Accepted 2021-10-11 